\title{
Existence and Uniqueness of Positive and Nondecreasing Solutions for a Class of Singular Fractional Boundary Value Problems
}

\author{
J. Caballero Mena, J. Harjani, and K. Sadarangani \\ Departamento de Matemáticas, Universidad de Las Palmas de Gran Canaria, Campus de Tafira Baja, \\ 35017 Las Palmas de Gran Canaria, Spain \\ Correspondence should be addressed to K. Sadarangani, ksadaran@dma.ulpgc.es
}

Received 24 April 2009; Accepted 14 June 2009

Recommended by Juan José Nieto

We establish the existence and uniqueness of a positive and nondecreasing solution to a singular boundary value problem of a class of nonlinear fractional differential equation. Our analysis relies on a fixed point theorem in partially ordered sets.

Copyright @ 2009 J. Caballero Mena et al. This is an open access article distributed under the Creative Commons Attribution License, which permits unrestricted use, distribution, and reproduction in any medium, provided the original work is properly cited.

\section{Introduction}

Many papers and books on fractional differential equations have appeared recently. Most of them are devoted to the solvability of the linear fractional equation in terms of a special function (see, e.g., [1,2]) and to problems of analyticity in the complex domain [3]. Moreover, Delbosco and Rodino [4] considered the existence of a solution for the nonlinear fractional differential equation $D_{0^{+}}^{\alpha} u=f(t, u)$, where $0<\alpha<1$ and $f:[0, a] \times \mathbb{R} \rightarrow \mathbb{R}, 0<a \leq+\infty$ is a given continuous function in $(0, a) \times \mathbb{R}$. They obtained results for solutions by using the Schauder fixed point theorem and the Banach contraction principle. Recently, Zhang [5] considered the existence of positive solution for equation $D_{0^{+}}^{\alpha} u=f(t, u)$, where $0<\alpha<1$ and $f:[0,1] \times[0, \infty) \rightarrow[0, \infty)$ is a given continuous function by using the sub- and supersolution methods.

In this paper, we discuss the existence and uniqueness of a positive and nondecreasing solution to boundary-value problem of the nonlinear fractional differential equation

$$
\begin{gathered}
D_{0^{+}}^{\alpha} u(t)+f(t, u(t))=0, \quad 0<t<1, \\
u(0)=u^{\prime}(1)=u^{\prime \prime}(0)=0,
\end{gathered}
$$


where $2<\alpha \leq 3, D_{0^{+}}^{\alpha}$ is the Caputo's differentiation and $f:(0,1] \times[0, \infty) \rightarrow[0, \infty)$ with $\lim _{t \rightarrow 0^{+}} f(t,-)=\infty$ (i.e., $f$ is singular at $t=0$ ).

Note that this problem was considered in [6] where the authors proved the existence of one positive solution for (1.1) by using Krasnoselskii's fixed point theorem and nonlinear alternative of Leray-Schauder type in a cone and assuming certain hypotheses on the function $f$. In [6] the uniqueness of the solution is not treated.

In this paper we will prove the existence and uniqueness of a positive and nondecreasing solution for the problem (1.1) by using a fixed point theorem in partially ordered sets.

Existence of fixed point in partially ordered sets has been considered recently in [7-12]. This work is inspired in the papers $[6,8]$.

For existence theorems for fractional differential equation and applications, we refer to the survey [13]. Concerning the definitions and basic properties we refer the reader to [14].

Recently, some existence results for fractional boundary value problem have appeared in the literature (see, e.g., [15-17]).

\section{Preliminaries and Previous Results}

For the convenience of the reader, we present here some notations and lemmas that will be used in the proofs of our main results.

Definition 2.1. The Riemman-Liouville fractional integral of order $\alpha>0$ of a function $f$ : $(0, \infty) \rightarrow \mathbb{R}$ is given by

$$
I_{0^{+}}^{\alpha} f(t)=\frac{1}{\Gamma(\alpha)} \int_{0}^{t}(t-s)^{\alpha-1} f(s) d s
$$

provided that the right-hand side is pointwise defined on $(0, \infty)$.

Definition 2.2. The Caputo fractional derivative of order $\alpha>0$ of a continuous function $f$ : $(0, \infty) \rightarrow \mathbb{R}$ is given by

$$
D_{0^{+}}^{\alpha} f(t)=\frac{1}{\Gamma(n-\alpha)} \int_{0}^{t} \frac{f^{(n)}(s)}{(t-s)^{\alpha-n+1}} d s,
$$

where $n-1<\alpha \leq n$, provided that the right-hand side is pointwise defined on $(0, \infty)$.

The following lemmas appear in [14].

Lemma 2.3. Let $n-1<\alpha \leq n, u \in C^{(n)}[0,1]$. Then

$$
I_{0^{+}}^{\alpha} D_{0^{+}}^{\alpha} u(t)=u(t)-c_{1}-c_{2} t-\cdots-c_{n} t^{n-1},
$$

where $c_{i} \in \mathbb{R}, i=1,2, \ldots, n$. 
Lemma 2.4. The relation

$$
I_{0^{+}}^{\alpha} I_{0^{+}}^{\beta} \varphi=I_{0^{+}}^{\alpha+\beta} \varphi
$$

is valid when $\operatorname{Re} \beta>0, \operatorname{Re}(\alpha+\beta)>0, \varphi(x) \in L^{1}(0, b)$.

The following lemmas appear in [6].

Lemma 2.5. Given $f \in C[0,1]$ and $2<\alpha \leq 3$, the unique solution of

$$
\begin{gathered}
D_{0^{+}}^{\alpha} u(t)+f(t)=0, \quad 0<t<1, \\
u(0)=u^{\prime}(1)=u^{\prime \prime}(0)=0,
\end{gathered}
$$

is given by

$$
u(t)=\int_{0}^{1} G(t, s) f(s) d s
$$

where

$$
G(t, s)= \begin{cases}\frac{(\alpha-1) t(1-s)^{\alpha-2}-(t-s)^{\alpha-1}}{\Gamma(\alpha)}, & 0 \leq s \leq t \leq 1 \\ \frac{t(1-s)^{\alpha-2}}{\Gamma(\alpha-1)}, & 0 \leq t \leq s \leq 1\end{cases}
$$

Remark 2.6. Note that $G(t, s)>0$ for $t \neq 0$ and $G(0, s)=0$ (see [6]).

Lemma 2.7. Let $0<\sigma<1,2<\alpha \leq 3$ and $F:(0,1] \rightarrow \mathbb{R}$ is a continuous function with $\lim _{t \rightarrow 0^{+}} F(t)=\infty$. Suppose that $t^{\sigma} F(t)$ is a continuous function on $[0,1]$. Then the function defined by

$$
H(t)=\int_{0}^{1} G(t, s) F(s) d s
$$

is continuous on $[0,1]$, where $G(t, s)$ is the Green function defined in Lemma 2.5 .

Now, we present some results about the fixed point theorems which we will use later. These results appear in [8].

Theorem 2.8. Let $(X, \leq)$ be a partially ordered set and suppose that there exists a metric $d$ in $X$ such that $(X, d)$ is a complete metric space. Assume that $X$ satisfies the following condition: if $\left\{x_{n}\right\}$ is a non decreasing sequence in $X$ such that $x_{n} \rightarrow x$ then $x_{n} \leq x$ for all $n \in \mathbb{N}$. Let $T: X \rightarrow X$ be a nondecreasing mapping such that

$$
d(T x, T y) \leq d(x, y)-\psi(d(x, y)), \text { for } x \geq y,
$$


where $\psi:[0, \infty) \rightarrow[0, \infty)$ is continuous and nondecreasing function such that $\psi$ is positive in $(0, \infty), \psi(0)=0$ and $\lim _{t \rightarrow \infty} \psi(t)=\infty$. If there exists $x_{0} \in X$ with $x_{0} \leq T\left(x_{0}\right)$ then $T$ has a fixed point.

If we consider that $(X, \leq)$ satisfies the following condition:

for $x, y \in X$ there exists $z \in X$ which is comparable to $x$ and $y$,

then we have the following theorem [8].

Theorem 2.9. Adding condition (2.10) to the hypotheses of Theorem 2.8 one obtains uniqueness of the fixed point of $f$.

In our considerations, we will work in the Banach space $C[0,1]=\{x:[0,1] \rightarrow$ $\mathbb{R}$, continuous $\}$ with the standard norm $\|x\|=\max _{0 \leq t \leq 1}|x(t)|$.

Note that this space can be equipped with a partial order given by

$$
x, y \in C[0,1], \quad x \leq y \Longleftrightarrow x(t) \leq y(t), \quad \text { for } t \in[0,1] .
$$

In $[10]$ it is proved that $(C[0,1], \leq)$ with the classic metric given by

$$
d(x, y)=\max _{0 \leq t \leq 1}\{|x(t)-y(t)|\}
$$

satisfies condition (2) of Theorem 2.8. Moreover, for $x, y \in C[0,1]$, as the function $\max \{x, y\}$ is continuous in $[0,1],(C[0,1], \leq)$ satisfies condition $(2.10)$.

\section{Main Result}

Theorem 3.1. Let $0<\sigma<1,2<\alpha \leq 3, f:(0,1] \times(0, \infty) \rightarrow[0, \infty)$ is continuous and $\lim _{t \rightarrow 0^{+}} f(t,-)=\infty, t^{\sigma} f(t, y)$ is a continuous function on $[0,1] \times[0, \infty)$. Assume that there exists $0<\lambda \leq \Gamma(\alpha-\sigma) / \Gamma(1-\sigma)$ such that for $x, y \in[0, \infty)$ with $y \geq x$ and $t \in[0,1]$

$$
0 \leq t^{\sigma}(f(t, y)-f(t, x)) \leq \lambda \cdot \ln (y-x+1)
$$

Then one's problem (1.1) has an unique nonnegative solution.

Proof. Consider the cone

$$
P=\{u \in C[0,1]: u(t) \geq 0\} .
$$

Note that, as $P$ is a closed set of $C[0,1], P$ is a complete metric space. 
Now, for $u \in P$ we define the operator $T$ by

$$
(T u)(t)=\int_{0}^{1} G(t, s) f(s, u(s)) d s
$$

By Lemma 2.7, $T u \in C[0,1]$. Moreover, taking into account Remark 2.6 and as $t^{\sigma} f(t, y) \geq 0$ for $(t, y) \in[0,1] \times[0, \infty)$ by hypothesis, we get

$$
(T u)(t)=\int_{0}^{1} G(t, s) s^{-\sigma} s^{\sigma} f(s, u(s)) d s \geq 0 .
$$

Hence, $T(P) \subset P$.

In what follows we check that hypotheses in Theorems 2.8 and 2.9 are satisfied.

Firstly, the operator $T$ is nondecreasing since, by hypothesis, for $u \geq v$

$$
\begin{aligned}
(T u)(t) & =\int_{0}^{1} G(t, s) f(s, u(s)) d s \\
& =\int_{0}^{1} G(t, s) s^{-\sigma} s^{\sigma} f(s, u(s)) d s \\
& \geq \int_{0}^{1} G(t, s) s^{-\sigma} s^{\sigma} f(s, v(s)) d s=(T v)(t) .
\end{aligned}
$$

Besides, for $u \geq v$

$$
\begin{aligned}
d(T u, T v) & =\max _{t \in[0,1]}|(T u)(t)-(T v)(t)| \\
& =\max _{t \in[0,1]}((T u)(t)-(T v)(t))=\max _{t \in[0,1]}\left[\int_{0}^{1} G(t, s)(f(s, u(s))-f(s, v(s))) d s\right] \\
& =\max _{t \in[0,1]}\left[\int_{0}^{1} G(t, s) s^{-\sigma} s^{\sigma}(f(s, u(s))-f(s, v(s))) d s\right] \\
& \leq \max _{t \in[0,1]}\left[\int_{0}^{1} G(t, s) s^{-\sigma} \lambda \cdot \ln (u(s)-v(s)+1) d s\right]
\end{aligned}
$$

As the function $\varphi(x)=\ln (x+1)$ is nondecreasing then, for $u \geq v$,

$$
\ln (u(s)-v(s)+1) \leq \ln (\|u-v\|+1)
$$


and from last inequality we get

$$
\begin{aligned}
& d(T u, T v) \leq \max _{t \in[0,1]}\left[\int_{0}^{1} G(t, s) s^{-\sigma} \mathcal{\lambda} \cdot \ln (u(s)-v(s)+1) d s\right] \\
& \leq \lambda \cdot \ln (\|u-v\|+1) \cdot \max _{t \in[0,1]} \int_{0}^{1} G(t, s) s^{-\sigma} d s \\
& =\lambda \cdot \ln (\|u-v\|+1) \\
& \cdot \max _{t \in[0,1]}\left[\int_{0}^{t} \frac{(\alpha-1) t(1-s)^{\alpha-2}-(t-s)^{\alpha-1}}{\Gamma(\alpha)} s^{-\sigma} d s+\int_{t}^{1} \frac{t(1-s)^{\alpha-2}}{\Gamma(\alpha-1)} s^{-\sigma} d s\right] \\
& \leq \lambda \cdot \ln (\|u-v\|+1) \\
& \cdot \max _{t \in[0,1]}\left[\int_{0}^{t} \frac{(\alpha-1) t(1-s)^{\alpha-2}}{\Gamma(\alpha)} s^{-\sigma} d s+\int_{t}^{1} \frac{t(1-s)^{\alpha-2} \cdot s^{-\sigma}}{\Gamma(\alpha-1)} d s\right] \\
& \leq \lambda \cdot \ln (\|u-v\|+1) \\
& \cdot \max _{t \in[0,1]}\left[\int_{0}^{t} \frac{(\alpha-1)(1-s)^{\alpha-2}}{\Gamma(\alpha)} s^{-\sigma} d s+\int_{t}^{1} \frac{(1-s)^{\alpha-2} \cdot s^{-\sigma}}{\Gamma(\alpha-1)} d s\right] \\
& =\lambda \cdot \ln (\|u-v\|+1) \cdot \max _{t \in[0,1]}\left[\int_{0}^{t} \frac{(1-s)^{\alpha-2} s^{-\sigma}}{\Gamma(\alpha-1)} d s+\int_{t}^{1} \frac{(1-s)^{\alpha-2} s^{-\sigma}}{\Gamma(\alpha-1)} d s\right] \\
& =\frac{\lambda \cdot \ln (\|u-v\|+1)}{\Gamma(\alpha-1)} \cdot \max _{t \in[0,1]}\left[\int_{0}^{1}(1-s)^{\alpha-2} s^{-\sigma} d s\right] \\
& =\frac{\lambda \cdot \ln (\|u-v\|+1)}{\Gamma(\alpha-1)} \cdot \int_{0}^{1}(1-s)^{\alpha-2} s^{-\sigma} d s \\
& =\frac{\lambda \cdot \ln (\|u-v\|+1)}{\Gamma(\alpha-1)} \cdot \beta(1-\sigma, \alpha-1) \\
& =\frac{\lambda \cdot \ln (\|u-v\|+1)}{\Gamma(\alpha-1)} \cdot \frac{\Gamma(1-\sigma) \cdot \Gamma(\alpha-1)}{\Gamma(\alpha-\sigma)} \\
& =\lambda \cdot \ln (\|u-v\|+1) \cdot \frac{\Gamma(1-\sigma)}{\Gamma(\alpha-\sigma)} \leq \frac{\Gamma(\alpha-\sigma)}{\Gamma(1-\sigma)} \cdot \lambda \cdot \ln (\|u-v\|+1) \cdot \frac{\Gamma(1-\sigma)}{\Gamma(\alpha-\sigma)} \\
& =\ln (\|u-v\|+1)=\|u-v\|-(\|u-v\|-\ln (\|u-v\|+1)) \text {. }
\end{aligned}
$$

Put $\psi(x)=x-\ln (x+1)$. Obviously, $\psi:[0, \infty) \rightarrow[0, \infty)$ is continuous, nondecreasing, positive in $(0, \infty), \psi(0)=0$ and $\lim _{x \rightarrow \infty} \psi(x)=\infty$.

Thus, for $u \geq v$

$$
d(T u, T v) \leq d(u, v)-\psi(d(u, v))
$$


Finally, take into account that for the zero function, $0 \leq T 0$, by Theorem 2.8 our problem (1.1) has at least one nonnegative solution. Moreover, this solution is unique since $(P, \leq)$ satisfies condition (2.10) (see comments at the beginning of this section) and Theorem 2.9.

Remark 3.2. In [6, lemma 3.2] it is proved that $T: P \rightarrow P$ is completely continuous and Schauder fixed point theorem gives us the existence of a solution to our problem (1.1).

In the sequel we present an example which illustrates Theorem 3.1.

Example 3.3. Consider the fractional differential equation (this example is inspired in [6])

$$
\begin{gathered}
D_{0^{+}}^{5 / 2} u(t)+\frac{(t-1 / 2)^{2} \ln (2+u(t))}{\sqrt{t}}=0, \quad 0<t<1 \\
u(0)=u^{\prime}(1)=u^{\prime \prime}(0)=0
\end{gathered}
$$

In this case, $f(t, u)=(t-1 / 2)^{2} \ln (2+u(t)) / \sqrt{t}$ for $(t, u) \in(0,1] \times[0, \infty)$. Note that $f$ is continuous in $(0,1] \times[0, \infty)$ and $\lim _{t \rightarrow 0^{+}} f(t,-)=\infty$. Moreover, for $u \geq v$ and $t \in[0,1]$ we have

$$
0 \leq \sqrt{t}\left(\left(t-\frac{1}{2}\right)^{2} \ln (2+u)-\left(t-\frac{1}{2}\right)^{2} \ln (2+v)\right)
$$

because $g(x)=\ln (x+2)$ is nondecreasing on $[0, \infty)$, and

$$
\begin{aligned}
& \sqrt{t}\left(\left(t-\frac{1}{2}\right)^{2} \ln (2+u)-\left(t-\frac{1}{2}\right)^{2} \ln (2+v)\right) \\
& \quad=\sqrt{t} \cdot\left(t-\frac{1}{2}\right)^{2}[\ln (2+u)-\ln (2+v)] \\
& \quad=\sqrt{(t)}\left(t-\frac{1}{2}\right)^{2}\left[\ln \left(\frac{2+u}{2+v}\right)\right]=\sqrt{t}\left(t-\frac{1}{2}\right)^{2} \ln \left(\frac{2+v+u-v}{2+v}\right) \\
& \leq\left(\frac{1}{2}\right)^{2} \ln (1+u-v) .
\end{aligned}
$$

Note that $\Gamma(\alpha-\sigma) / \Gamma(1-\sigma)=\Gamma(5 / 2-1 / 2) / \Gamma(1-1 / 2)=\Gamma(2) / \Gamma(1 / 2)=1 / \sqrt{\pi} \geq 1 / 4$.

Theorem 3.1 give us that our fractional differential (3.10) has an unique nonnegative solution.

This example give us uniqueness of the solution for the fractional differential equation appearing in [6] in the particular case $\sigma=1 / 2$ and $\alpha=5 / 2$

Remark 3.4. Note that our Theorem 3.1 works if the condition (3.1) is changed by, for $x, y \in$ $[0, \infty)$ with $y \geq x$ and $t \in[0,1]$

$$
0 \leq t^{\sigma}(f(t, y)-f(t, x)) \leq \lambda \cdot \psi(y-x)
$$


where $\psi:[0, \infty) \rightarrow[0, \infty)$ is continuous and $\varphi(x)=x-\psi(x)$ satisfies

(a) $\varphi:[0, \infty) \rightarrow[0, \infty)$ and nondecreasing;

(b) $\varphi(0)=0$;

(c) $\varphi$ is positive in $(0, \infty)$;

(d) $\lim _{x \rightarrow \infty} \varphi(x)=\infty$.

Examples of such functions are $\psi(x)=\operatorname{arctg} x$ and $\psi(x)=x /(1+x)$.

Remark 3.5. Note that the Green function $G(t, s)$ is strictly increasing in the first variable in the interval $(0,1)$. In fact, for $s$ fixed we have the following cases

Case 1. For $t_{1}, t_{2} \leq s$ and $t_{1}<t_{2}$ as, in this case,

$$
G(t, s)=\frac{t(1-s)^{\alpha-2}}{\Gamma(\alpha-1)}
$$

It is trivial that

$$
G\left(t_{1}, s\right)=\frac{t_{1}(1-s)^{\alpha-2}}{\Gamma(\alpha-1)}<\frac{t_{2}(1-s)^{\alpha-2}}{\Gamma(\alpha-1)}=G\left(t_{2}, s\right)
$$

Case 2. For $t_{1} \leq s \leq t_{2}$ and $t_{1}<t_{2}$, we have

$$
\begin{aligned}
G\left(t_{2}, s\right)-G\left(t_{1}, s\right) & =\left[\frac{(\alpha-1) t_{2}(1-s)^{\alpha-2}}{\Gamma(\alpha)}-\frac{\left(t_{2}-s\right)^{\alpha-1}}{\Gamma(\alpha)}\right]-\left[\frac{t_{1}(1-s)^{\alpha-2}}{\Gamma(\alpha-1)}\right] \\
& =\frac{t_{2}(1-s)^{\alpha-2}-t_{1}(1-s)^{\alpha-2}}{\Gamma(\alpha-1)}-\frac{\left(t_{2}-s\right)^{\alpha-1}}{\Gamma(\alpha)} \\
& >\frac{\left(t_{2}-t_{1}\right)(1-s)^{\alpha-2}}{\Gamma(\alpha-1)}-\frac{\left(t_{2}-s\right)^{\alpha-1}}{\Gamma(\alpha-1)} \\
& =\frac{\left(t_{2}-t_{1}\right)(1-s)^{\alpha-2}}{\Gamma(\alpha-1)}-\frac{\left(t_{2}-s\right)\left(t_{2}-s\right)^{\alpha-2}}{\Gamma(\alpha-1)} .
\end{aligned}
$$

Now, $t_{2}-t_{1} \geq\left(t_{2}-s\right)$ and $(1-s) \geq\left(t_{2}-s\right)$ then

$$
\frac{\left(t_{2}-t_{1}\right)(1-s)^{\alpha-2}}{\Gamma(\alpha-1)}>\frac{\left(t_{2}-s\right)\left(t_{2}-s\right)^{\alpha-2}}{\Gamma(\alpha-1)} .
$$

Hence, taking into account the last inequality and (3.16), we obtain $G\left(t_{1}, s\right)<G\left(t_{2}, s\right)$.

Case 3. For $s \leq t_{1}, t_{2}$ and $t_{1}<t_{2}<1$, we have

$$
\frac{\partial G}{\partial t}=\frac{(\alpha-1)(1-s)^{\alpha-2}-(\alpha-1)(1-s)^{\alpha-2}}{\Gamma(\alpha)}=\frac{\alpha-1}{\Gamma(\alpha)}\left((1-s)^{\alpha-2}-(t-s)^{\alpha-2}\right),
$$


and, as $(1-s)^{\alpha-2}>(t-s)^{\alpha-2}$ for $t \in[0,1)$, it can be deduced that $\partial G / \partial t>0$ and consequently, $G\left(t_{2}, s\right)>G\left(t_{1}, s\right)$.

This completes the proof.

Remark 3.5 gives us the following theorem which is a better result than that $[6$, Theorem 3.3] because the solution of our problem (1.1) is positive in $(0,1)$ and strictly increasing.

Theorem 3.6. Under assumptions of Theorem 3.1, our problem (1.1) has a unique nonnegative and strictly increasing solution.

Proof. By Theorem 3.1 we obtain that the problem (1.1) has an unique solution $u(t) \in C[0,1]$ with $u(t) \geq 0$. Now, we will prove that this solution is a strictly increasing function. Let us take $t_{2}, t_{1} \in[0,1]$ with $t_{1}<t_{2}$, then

$$
u\left(t_{2}\right)-u\left(t_{1}\right)=(T u)\left(t_{2}\right)-(T u)\left(t_{1}\right)=\int_{0}^{1}\left(G\left(t_{2}, s\right)-G\left(t_{1}, s\right)\right) f(s, u(s)) d s .
$$

Taking into account Remark 3.4 and the fact that $f \geq 0$, we get $u\left(t_{2}\right)-u\left(t_{1}\right) \geq 0$.

Now, if we suppose that $u\left(t_{2}\right)-u\left(t_{1}\right)=0$ then $\int_{0}^{1}\left(G\left(t_{2}, s\right)-G\left(t_{1}, s\right)\right) f(s, u(s)) d s=0$ and as, $G\left(t_{2}, s\right)-G\left(t_{1}, s\right)>0$ we deduce that $f(s, u(s))=0$ a.e.

On the other hand, if $f(s, u(s))=0$ a.e. then

$$
u(t)=\int_{0}^{1} G(t, s) f(s, u(s)) d s=0 \quad \text { for } t \in[0,1]
$$

Now, as $\lim _{t \rightarrow 0^{+}} f(t, 0)=\infty$, then for $M>0$ there exists $\delta>0$ such that for $s \in[0,1]$ with $0<s<\delta$ we get $f(s, 0)>M$. Observe that $(0, \delta) \subset\{s \in[0,1]: f(s, u(s))>M\}$, consequently,

$$
\delta=\mu((0, \delta)) \leq \mu(\{s \in[0,1]: f(s, u(s))>M\})
$$

and this contradicts that $f(s, u(s))=0$ a.e.

Thus, $u\left(t_{2}\right)-u\left(t_{1}\right)>0$ for $t_{2}, t_{1} \in[0,1]$ with $t_{2}>t_{1}$. Finally, as $u(0)=$ $\int_{0}^{1} G(0, s) f(s, u(s)) d s=0$ we have that $0<u(t)$ for $t \neq 0$.

\section{Acknowledgment}

This research was partially supported by "Ministerio de Educación y Ciencia" Project MTM $2007 / 65706$.

\section{References}

[1] L. M. B. C. Campos, "On the solution of some simple fractional differential equations," International Journal of Mathematics and Mathematical Sciences, vol. 13, no. 3, pp. 481-496, 1990.

[2] K. S. Miller and B. Ross, An Introduction to the Fractional Calculus and Fractional Differential Equations, A Wiley-Interscience Publication, John Wiley \& Sons, New York, NY, USA, 1993. 
[3] Y. Ling and S. Ding, "A class of analytic functions defined by fractional derivation," Journal of Mathematical Analysis and Applications, vol. 186, no. 2, pp. 504-513, 1994.

[4] D. Delbosco and L. Rodino, "Existence and uniqueness for a nonlinear fractional differential equation," Journal of Mathematical Analysis and Applications, vol. 204, no. 2, pp. 609-625, 1996.

[5] S. Zhang, "The existence of a positive solution for a nonlinear fractional differential equation," Journal of Mathematical Analysis and Applications, vol. 252, no. 2, pp. 804-812, 2000.

[6] T. Qiu and Z. Bai, "Existence of positive solutions for singular fractional differential equations," Electronic Journal of Differential Equations, vol. 2008, no. 146, pp. 1-9, 2008.

[7] L. Ćirić, N. Cakić, M. Rajović, and J. S. Ume, "Monotone generalized nonlinear contractions in partially ordered metric spaces," Fixed Point Theory and Applications, vol. 2008, Article ID 131294, 11 pages, 2008.

[8] J. Harjani and K. Sadarangani, "Fixed point theorems for weakly contractive mappings in partially ordered sets," Nonlinear Analysis: Theory, Methods \& Applications, vol. 71, no. 7-8, pp. 3403-3410, 2009.

[9] J. J. Nieto, R. L. Pouso, and R. Rodríguez-López, "Fixed point theorems in ordered abstract spaces," Proceedings of the American Mathematical Society, vol. 135, no. 8, pp. 2505-2517, 2007.

[10] J. J. Nieto and R. Rodríguez-López, "Contractive mapping theorems in partially ordered sets and applications to ordinary differential equations," Order, vol. 22, no. 3, pp. 223-239, 2005.

[11] J. J. Nieto and R. Rodríguez-López, "Existence and uniqueness of fixed point in partially ordered sets and applications to ordinary differential equations," Acta Mathematica Sinica, vol. 23, no. 12, pp. 2205-2212, 2007.

[12] D. O'Regan and A. Petruşel, "Fixed point theorems for generalized contractions in ordered metric spaces," Journal of Mathematical Analysis and Applications, vol. 341, no. 2, pp. 1241-1252, 2008.

[13] A. A. Kilbas and J. J. Trujillo, "Differential equations of fractional order: methods, results and problems-I," Applicable Analysis, vol. 78, no. 1-2, pp. 153-192, 2001.

[14] S. G. Samko, A. A. Kilbas, and O. I. Marichev, Fractional Integrals and Derivatives. Theory and Applications, Gordon and Breach Science, Yverdon, Switzerland, 1993.

[15] B. Ahmad and J. J. Nieto, "Existence results for nonlinear boundary value problems of fractional integrodifferential equations with integral boundary conditions," Boundary Value Problems, vol. 2009, Article ID 708576, 11 pages, 2009.

[16] M. Belmekki, J. J. Nieto, and R. Rodríguez-López, "Existence of periodic solution for a nonlinear fractional differential equation," Boundary Value Problems. In press.

[17] Y.-K. Chang and J. J. Nieto, "Some new existence results for fractional differential inclusions with boundary conditions," Mathematical and Computer Modelling, vol. 49, no. 3-4, pp. 605-609, 2009. 